\title{
Evidências de validade da Escala de Avaliação do Relacionamento
}

\author{
Validity evidences the Relationship \\ Assessment Scale
}

José Augusto Evangelho HERNANDEZ ${ }^{1}$

\begin{abstract}
Resumo
Este estudo, de caráter exploratório e confirmatório, buscou evidências de validade para a Escala de Avaliação do Relacionamento de Hendrick. No primeiro estudo, participaram 201 pessoas de ambos os sexos que coabitavam com seus parceiros na cidade de João Pessoa, Paraíba. A análise fatorial exploratória para componentes principais dos escores da escala extraiu solução inicial com um fator responsável pela explicação de $61 \%$ da variância total e com coeficiente alfa de 0,89. No segundo estudo, participaram 538 pessoas envolvidas em diversos tipos de relacionamentos amorosos da cidade de Porto Alegre, Rio Grande do Sul. Uma análise fatorial confirmatória (GFI = 0,97, razão CMIN/ $\mathrm{DF}=3,62$ ) indicou a adequação do modelo. Análises das relações dessa escala com outras medidas de satisfação no relacionamento revelaram correlações estatísticas significativas que variaram de moderadas altas a fortes. Esses resultados evidenciaram a validade fatorial, convergente e consistência interna para escores oriundos de diferentes culturas.
\end{abstract}

Palavras-chave: Satisfação conjugal; Satisfação no relacionamento; Psicometria.

\begin{abstract}
This exploratory and confirmatory study sought evidence for the validity of the Relationship Assessment Scale of Hendrick. A total of 201 people of both sexes that lived with their partners in the city of João Pessoa, Paraíba, participated in the first study. The exploratory factor analysis for the principal components of the scale scores extracted an initial solution with one factor responsible for explaining $61 \%$ of the total variance and a coefficient alpha of 0.89 . A total of 538 people involved in different types of affective relationships from Porto Alegre, Rio Grande do Sul, participated in the second study. The confirmatory factor analysis (GFI =0.97, CMINIDF ratio $=3.62)$ indicated the appropriateness of the model. Analysis of the correlations of this scale with other measures of relationship satisfaction revealed statistically significant correlations, which ranged from high-moderate to high. These findings showed factorial validity, convergent and internal consistency for scores from different cultures.
\end{abstract}

Keywords: Marital satisfaction; Relationship satisfaction; Psychometrics.

Se as pessoas estão casadas, coabitam ou têm um relacionamento estável, e se os casais são heterossexuais ou homossexuais, as questões de satisfação são igualmente importantes. A satisfação

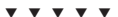

${ }^{1}$ Universidade do Estado do Rio de Janeiro, Instituto de Psicologia, Departamento de Fundamentos da Psicologia. R. São Francisco Xavier, 524, $10^{\circ}$ andar, 20550-013, Rio de Janeiro, RJ, Brasil. E-mail: <hernandez.uerj@gmail.com>.
} 
pode ter fortes implicações para os parceiros permanecerem juntos ou romperem seus relacionamentos (S.S. Hendrick, Hendrick, \& Adler, 1988), sendo assim, é um construto relevante e muito discutido na Psicologia. A presente investigação procurou fornecer evidências de validade para a Escala de Avaliação do Relacionamento (EAR) de Hendrick (1988) com dados de brasileiros e examinou a estrutura fatorial e a consistência interna desse instrumento bem como sua associação com outras medidas de satisfação nas relações amorosas.

Berscheid (2010) afirmou que, nas últimas décadas, a pesquisa sobre satisfação e estabilidade conjugal aumentou em quantidade, sofisticação metodológica e analítica. Muito disso foi estimulado pelo resultado do estudo longitudinal de Burgess e Wallin (1944). Após duas décadas de casamento, a satisfação dos cônjuges tinha declinado, a intimidade e as atividades compartilhadas, incluindo a relação sexual, tinham diminuído. Posteriormente, estudos transversais corroboraram o declínio da satisfação ao longo do tempo, com a ressalva de que um ligeiro aumento foi visto em casamentos de longa duração, a chamada curva U. Contudo, estudos longitudinais revelaram que esse aumento de satisfação nos casamentos de longa duração era uma ilusão produzida pela metodologia transversal. Em geral, casais altamente insatisfeitos não aparecem no longo prazo porque já estão divorciados ou separados.

As explicações para o declínio focavam exclusivamente os conflitos e os sentimentos negativos que os acompanhavam. Em decorrência disso, as terapias para casamentos estressados se concentraram na resolução de conflitos e nas habilidades de comunicação. Porém, a suposição de que o conflito é a única ou a principal causa da insatisfação conjugal passou a ser questionada. A duração e a felicidade dos relacionamentos parecem estar relacionadas também a comportamentos afetuosos e de apoio do que apenas à ausência de antagonismo e conflito (Huston, Caughlin, Houts, Smith, \& George, 2001).

Satisfação é apenas um dos diversos termos empregados para descrever uma porção de jul328 gamentos sobre um relacionamento íntimo. Quali- dade conjugal, ajustamento conjugal e sucesso conjugal também têm sido usados para denotar satisfação segundo S.S. Hendrick e Hendrick (1997).

Glenn (1990) diferenciou satisfação de felicidade, e ambas foram caracterizadas como índices individuais de qualidade conjugal. Entretanto, o autor empregou o termo ajustamento como índice de qualidade conjugal orientado ao relacionamento. Para S.S. Hendrick e Hendrick (1997), a satisfação engloba sentimentos subjetivos dos parceiros sobre seus relacionamentos, enquanto o ajustamento conjugal compreende comportamentos reais do relacionamento, que revelariam com precisão a forma como a relação funciona. Um casal poderia ter um bom relacionamento em termos comportamentais, os parceiros concordariam em estratégias da parentalidade, trabalhariam juntos sem conflito, organizariam sua vida financeira de forma eficaz e teriam carreiras produtivas, portanto estariam bem ajustados. Entretanto, se estivessem emocionalmente distantes, estariam insatisfeitos. De outro modo, um casal poderia discordar sobre os estilos da parentalidade, organizar erradamente sua vida financeira e estar levemente satisfeito com o trabalho, porém, se fazem amor com frequência, poderiam sentir-se emocionalmente conectados e satisfeitos com a relação. Os pesquisadores comentaram que, com as diferenças de definição da satisfação no relacionamento, vieram algumas controvérsias acerca das medidas. Algumas escalas medem a satisfação individual global e favorecem mais itens relacionais fundamentados comportamentalmente (Spanier, 1976), outras são muito longas e inclusivas.

Os investigadores e os clínicos do relacionamento podem discordar acerca das abordagens de satisfação no relacionamento, que poderão variar do individual ao relacional, do global ao específico e em relação ao estilo de medida, breve e global ou longo e específico. Contudo, a maioria deles concorda que abordagens diferentes não devem ser incluídas na mesma medida (Glenn, 1990).

A medida de satisfação também tem sido criticada por ser confundida como viés de desejabilidade social. Pensa-se que as pessoas podem responder perguntas sobre a relação amorosa com base no que acreditam que seja uma resposta social- 
mente apropriada ou socialmente desejável, no lugar das suas reais atitudes, convicções e emoções. Entretanto, Fowers, Applegate, Olson e Pomerantz (1994) e Russell e Wells (1992) mostraram que satisfação na relação amorosa e desejabilidade social são variáveis inteiramente independentes.

Satisfação é uma das principais áreas de avaliação no relacionamento, com numerosas medidas para avaliar sentimentos, pensamentos ou comportamentos dentro da relação conjugal. As mais populares medidas incluem os 15 itens do Teste de Ajustamento Conjugal (Locke \& Wallace, 1959), os 400 itens do Checklist de Observação do Cônjuge (Patterson, 1976), os 280 itens do Inventário de Satisfação Conjugal (Snyder, 1979) e os 32 itens da Escala de Ajustamento Diádico (EAD) de Spanier (1976). Embora todas essas medidas sejam respeitadas, muitas são excessivamente longas e, às vezes, inviáveis para a pesquisa e a clínica, e, além disso, são direcionadas exclusivamente para o relacionamento conjugal.

A variedade de relacionamentos na sociedade contemporânea levou Hendrick (1988) ao desenvolvimento de uma medida de satisfação breve e confiável que fosse aplicável aos muitos tipos de relações íntimas. A EAR é uma medida global, unidimensional e breve (sete itens), baseada nos cinco itens do Questionário de Avaliação Conjugal de Hendrick (1981). As mudanças nessa escala envolveram a substituição das palavras companheiro/a por parceiro/a e casamento por relacionamento, e foram incluídos dois itens de uma versão anterior da mesma escala.

Na construção e desenvolvimento da EAR, estudo I, segundo Hendrick (1988), participaram 125 sujeitos em relação amorosa. Foi executada uma análise fatorial da EAR que extraiu um único fator, que foi responsável pela explicação de $46 \%$ da variância total. A correlação interitem variou de 0,11 a 0,67; a correlação item-total, de 0,57 a 0,76. No estudo II, com 57 casais, Hendrick encontrou uma estrutura consistente como a anterior. A melhor solução extraiu um fator que explicou 57\% da variância total. A correlação média interitem foi 0,49. O alfa de Cronbach foi de 0,87. A EAR apresentou uma correlação estatística significativa $(r=0,80)$ com a EAD. As escalas foram efetivas em discriminar casais que estavam juntos daqueles que romperam. A pesquisadora concluiu que esses resultados pareciam muito aceitáveis para um instrumento de apenas sete itens.

Reexaminando o instrumento, S.S. Hendrick, Dicke e Hendrick (1998) encontraram correlação moderada para alta entre a EAR e outras medidas de satisfação conjugal, como a EAD, além de boa fidedignidade teste-reteste. Levaram-se em conta amostras de casais de diversas etnias e idades e casais em terapias de casal ou familiar. Os autores concluíram que a EAR é uma medida genérica de satisfação no relacionamento, psicometricamente sólida, apropriada, útil e prática para avaliação de relações amorosas numa ampla variedade de ambientes de pesquisa.

Em relação à satisfação conjugal no Brasil, Scorsolini-Comin e Santos (2010) realizaram uma revisão integrativa das publicações do período de 1970 a 2008 nas bases de dados Literatura Latino-Americana e do Caribe em Ciências da Saúde (Lilacs) e Scientific Electronic Library Online (SciELO). Foram recuperadas apenas dez publicações sobre satisfação em quase quatro décadas, o que revelou a escassez de estudos nacionais sobre o tema. Entre os poucos estudos recuperados estão aqueles que utilizaram instrumentos para medir a satisfação conjugal. A pesquisa de Magagnin et al. (2003) utilizou a EAD, de Spanier (1976), e a EAR de Hendrick (1988), em 41 casais primíparos nos primeiros meses de gestação. Norgren, Souza, Kaslow, Hammerschmidt e Sharlin (2004) mediram a satisfação conjugal de 30 casais paulistas de mais de 20 anos de casamento com a EAD. Perlin e Diniz (2005) investigaram casais casados em que ambos os parceiros trabalhavam no âmbito público e mensuraram sua satisfação através da EAD. Em todos esses trabalhos, os itens dos instrumentos usados foram, apenas, traduzidos para o português e ajustados por consenso aos conteúdos dos construtos.

O primeiro estudo psicométrico gerador de evidências de validade fatorial para a EAD (Spanier, 1976) com escores de brasileiros foi realizado por Hernandez (2008), mais de três décadas após a construção do instrumento, o que mostra que a 
exploração do tema é recente em nosso meio. Entretanto, a EAD é um dos instrumentos mais usados na pesquisa internacional, haja vista que Spanier, em 1985, já registrava 1 mil publicações, e Prouty, Markowski e Barnes (2000) noticiavam que esse número já estaria em torno de alguns milhares.

Wachelke, Andrade, Cruz, Faggiani, e Natividade (2004) construíram e validaram um instrumento brasileiro denominado Escala Fatorial de Satisfação em Relacionamento de Casal. Além disso, Andrade, Garcia e Cano (2009) investigaram um modelo preditivo de satisfação global com o relacionamento romântico e utilizaram a Escala de Satisfação Global com Relacionamento Amoroso de Rusbult (1983).

O presente estudo exploratório e confirmatório buscou gerar algumas evidências de validade relativas à estrutura e à consistência interna da EAR. Também foram examinadas as relações desse instrumento com outras duas medidas de satisfação no relacionamento amoroso: uma breve e outra extensa. Além disso, a escala foi examinada por dois estudos, com dados oriundos de duas regiões brasileiras que, certamente, têm características culturais diversas.

\section{Método Estudo I}

Foi recrutada uma amostra de conveniência de 201 pessoas - 97 do sexo masculino e 104 do sexo feminino -, da cidade de João Pessoa (PB). A idade dos sujeitos variou de 18 a 69 anos, com média de 34,5 e Desvio-Padrão (DP) de 10,53. 0 tempo de duração do relacionamento variou de um mês a 50 anos, média de 10,8 anos e DP de 9,38 anos. Todos estavam em relação amorosa, mediante casamento formal, civil e religioso ou união estável; 166 tinham filhos e 35 não os tinham.

Os participantes responderam a EAR de Hendrick (1988), instrumento breve de sete itens que fornece uma medida genérica e unidimensional de satisfação no relacionamento. Os itens são frases interrogativas, e os respondentes usaram uma escala tipo Likert de cinco pontos para registrar suas res- postas ao instrumento. Na contagem dos escores, os itens 4 e 7 necessitaram ser invertidos, pois medem a insatisfação no relacionamento. Essa versão brasileira da EAR foi produzida a partir da original norte-americana (Hendrick, 1988), usando-se o método de back translation, no qual participaram dois psicólogos bilíngues.

Os dados foram coletados em locais variados, públicos e privados. Em praças, parques, shoppings, em estabelecimentos de ensino superior, em reuniões de casais e nos domicílios particulares dos participantes. Em todos os casos, foram cumpridas as exigências relativas à ética em pesquisa com seres humanos conforme a Resolução CNS n 196/96. O estudo foi aprovado pelo Comitê de Ética em Pesquisa da Universidade do Estado do Rio de Janeiro, Protocolo $n^{\circ}$ 018/2011. Os participantes foram informados sobre os objetivos da pesquisa e assinaram um Termo de Consentimento Livre e Esclarecido.

Os dados coletados foram digitados e analisados no Statistical Package of Social Sciences (SPSS), versão 20, por meio das técnicas estatísticas descritivas, análise fatorial de componentes principais e coeficiente alfa de Cronbach para a verificação da estrutura e da consistência interna da EAR.

\section{Resultados Estudo I}

A adequação dos dados à análise fatorial foi verificada através do teste Kaiser-Meyer-Olkin, que forneceu um valor de 0,89, considerado como muito bom ajuste. $O$ teste de esfericidade de Bartlett apresentou os valores $\chi^{2}(21)=729,88 ; p<0,01$, que permitiram rejeitar a hipótese nula e confirmar a utilização do método de análise para os dados coletados.

A análise fatorial exploratória para componentes principais, baseada na extração de autovalores maiores do que 1,0, extraiu uma solução inicial com um único fator para a EAR. Esse fator com autovalor de 4,29 foi responsável pela explicação de $61,3 \%$ da variância total. As cargas fatoriais dos sete itens do instrumento podem ser observadas na Tabela 1. 
Tabela 1

Matriz Fatorial da Escala de Avaliação de Relacionamento: estudos atual e original

\begin{tabular}{|c|c|c|c|}
\hline & \multirow{2}{*}{$\frac{\text { Estudo atual }}{\text { I }}$} & \multicolumn{2}{|c|}{ Hendrick (1981) } \\
\hline & & I & $\|$ \\
\hline 1. O quanto sua (seu) parceira (o) satisfaz as suas necessidades? & 0,81 & 0,77 & 0,80 \\
\hline 2. Em geral,quanto você está satisfeito com seu relacionamento? & 0,86 & 0,79 & 0,88 \\
\hline 3. O seu relacionamento é melhor que as relações que você conhece? & 0,75 & 0,72 & 0,83 \\
\hline 4. Quantas vezes você deseja não ter começado seu relacionamento? & 0,75 & 0,67 & 0,67 \\
\hline 5. Quanto seu relacionamento atual satisfaz suas expectativas do início da relação? & 0,82 & 0,58 & 0,62 \\
\hline 6. O quanto você ama sua (seu) parceira (o)? & 0,80 & 0,66 & 0,65 \\
\hline 7. Quantos problemas existem no seu relacionamento? & 0,66 & 0,49 & 0,79 \\
\hline Autovalores & 4,29 & * & * \\
\hline Variância Explicada \% & 61,30 & 46,00 & 57,00 \\
\hline Alfas de Cronbach & 0,89 & * & 0,87 \\
\hline
\end{tabular}

Nota: *Dados não publicados pela autora.

Método de Extração: análise de componentes principais.

Tabela 2

Matriz de Correlação Interitem da Escala de Avaliação de Relacionamento

\begin{tabular}{|c|c|c|c|c|c|c|c|}
\hline Itens ${ }^{*}$ & s1 & s2 & s3 & s4 & s5 & s6 & s7 \\
\hline s1 & 1,00 & & & & & & \\
\hline$s 2$ & 0,66 & 1,00 & & & & & \\
\hline s3 & 0,47 & 0,58 & 1,00 & & & & \\
\hline s4 & 0,56 & 0,57 & 0,46 & 1,00 & & & \\
\hline s5 & 0,67 & 0,71 & 0,52 & 0,55 & 1,00 & & \\
\hline s6 & 0,60 & 0,65 & 0,64 & 0,51 & 0,56 & 1,00 & \\
\hline s7 & 0,46 & 0,50 & 0,46 & 0,48 & 0,44 & 0,35 & 1,00 \\
\hline
\end{tabular}

Nota: Itens da Escala de Avaliação do Relacionamento.

A análise da consistência interna da escala mostrou um coeficiente alfa de Cronbach de 0,89. A média de correlação interitem foi 0,54 , com um mínimo de 0,35 e um máximo de 0,71 (Tabela 2). A correlação item-total variou de 0,56 a 0,79.

Análises de variância não encontraram diferenças estatísticas significativas $(p<0,05)$ de satisfação no relacionamento entre homens e mulheres, contudo, entre as condições com filho e sem filho na relação, foi apurado que os casais sem filhos $(\mathrm{M}=4,2$; $\mathrm{DP}=0,65)$ relataram satisfação no relacionamento significativamente maior $(p=0,03)$ do que os casais com filhos $(M=3,9 ; D P=0,67)$. Além disso, não houve correlações significativas entre satisfação no relacionamento e idade ou tempo de duração da relação dos participantes.

\section{Método Estudo II}

Em Porto Alegre (RS), 496 pessoas - 287 do sexo feminino e 209 do masculino -, responderam à EAR. A idade dos respondentes variou de 18 a 63 anos, com média de 29,3 e DP =9,33. O tempo de duração do relacionamento variou de um mês a 30 anos, com média de 6,5 anos e DP $=7$ anos. Dos participantes, 182 estavam formalmente casados ou em união estável; 297 eram noivos ou namorados e 17 não informaram esse dado. Além disso, 191 pessoas residiam junto com o parceiro, 287 não e 18 pessoas não forneceram essa informação; 117 têm filho na relação, 360 não e dezenove participantes não forneceram esse dado. A escolaridade dos participantes ficou distribuída da seguinte 
maneira: ensino fundamental ( $n=10)$, ensino médio ( $n=35)$, ensino superior $(n=385)$ e pós-graduação $(n=65)$. Uma pessoa não registrou a escolaridade.

Os participantes responderam à EAR, antes descrita. Também foi solicitado aos indivíduos que respondessem a dois itens (alfa de Cronbach de $0,93)$ criados por Jemmott III, Ashby, e Lindenfeld (1989) com objetivo de obter uma medida geral breve de satisfação no relacionamento para comparar com a EAR. Para que os participantes respondessem a esses itens, foi usada uma escala tipo Likert de 5 pontos: 1 significava nada satisfeito, e 5, totalmente satisfeito.

Além disso, foi usada a EAD de Spanier (1976), na versão brasileira (Hernandez, 2008). Esse instrumento é composto por 32 itens que buscam representar o ajustamento conjugal por meio das dimensões consenso diádico, satisfação diádica, coesão diádica e expressão de afeto. O instrumento foi respondido por meio de uma escala tipo Likert variada, com 5, 6 e 7 pontos, em geral, significando "nunca" e os 5, 6 ou 7 significando "todo o tempo", e dois itens (29 e 30) dicotômicos ("sim" ou "não"). O escore total da escala pode variar de 0 a 151 pontos e é obtido pela soma dos escores nos fatores: consenso (de 0 a 65), satisfação (de 0 a 50), coesão (de 0 a 24) e expressão de afeto (de 0 a 12). Alguns itens contêm afirmações positivas, e outros, negativas, tendo sido os escores desses últimos revertidos antes do cálculo da pontuação. Os indivíduos que obtiverem 101 pontos ou menos devem ser classificados como desajustados ou em sofrimento no relacionamento, e os que alcançarem 102 pontos ou mais, como ajustados (Spanier, 1976). Os coeficientes alfas de Cronbach calculados para a EAD total, subescalas consenso, satisfação, coesão e expressão de afeto foram de 0,93, 0,86, $0,86,0,76$ e 0,62 respectivamente (Hernandez, 2008).

Os procedimentos de coleta de dados foram os mesmos adotados no estudo 1. Para análise, os dados foram digitados e analisados no SPSS e no módulo AMOS Graphics, através de estatísticas descritivas e Análise Fatorial Confirmatória. Na

332 testagem de ajuste do modelo proposto, foram analisados os seguintes índices: Qui-quadrado $\left(\chi^{2}\right)$, Comparative Fit Index (CFl, Índice de Ajuste Comparativo), Root Mean Square Error of Approximation (RMSEA, Raiz Quadrada Média do Erro de Aproximação), Normed Fit Index (NFI, Índice de Ajuste Normalizado) e Goodness of Fit Index (GFI, Índice de Qualidade do Ajuste), conforme Boomsma (2000); Byrne, (2010); Hair, Anderson, Tatham e Black (2005) e McDonald e Ho (2002). Foi utilizado o coeficiente alfa de Cronbach para verificação da consistência interna da EAR, e o coeficiente de correlação de Pearson para examinar as relações dessa escala com outros instrumentos similares e possíveis evidências de validade convergente.

\section{Resultados Estudo II}

Na avaliação da normalidade dos escores dos sete itens da EAR, todos apresentaram leve assimetria negativa e curtose positiva, o que indica uma distribuiç̧ão moderadamente anormal. Em consequência, na execução da análise fatorial confirmatória, foi usado o método de estimação Máxima Verossimilhança. O modelo testado pode ser observado na Figura 1.

O Qui-quadrado, considerado uma das principais medidas de ajuste global do modelo, foi 50767 para 14 graus de liberdade com nível de significância menor do que 0,01. Esses valores

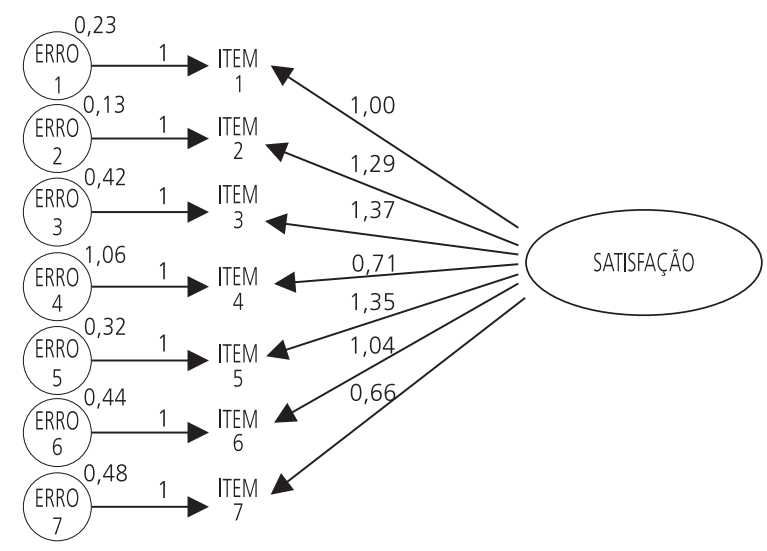

Figura 1. Modelo hipotético da Escala de Avaliação de Relacionamento testado com as estimativas não padronizadas. 
indicam que as matrizes dos dados observados diferem significativamente das estimadas. No entanto, como o $\chi^{2}$ é muito sensível a amostras grandes (maiores do que 200), situação do estudo atual $(n=537)$, recomenda-se a utilização da razão $\chi^{2} / g L$ como alternativa. Esse índice foi 3,6, que repre-senta a diferença entre as matrizes de dados e a estimada, portanto quanto menor o valor, menor a diferença. Critérios mais liberais consideram o valor 5,0 como limite superior máximo tolerado para o índice (Fife-Schaw, 2010).

A Root Mean Square Error of Approximation, medida da qualidade de ajuste se o modelo fosse estimado na população, foi 0,06. O intervalo estimado variou de 0,04 a 0,09, com 90\% de confiança. Segundo Hancock e Freeman (2001), o valor desse índice deve ser menor do que 0,08, representando uma pequena discrepância das matrizes em relação à população.

A Root Mean Square Residual (RMR, Raiz Quadrada Média Residual) foi 0,02, o que revela que os resíduos são reduzidos e denota que o modelo testado é plausível, ou seja, as variâncias e covariâncias da amostra pouco diferem das variâncias e covariâncias estimadas. Os resíduos representam a discrepância entre o modelo hipotetizado e os dados observados (Byrne, 2010).

O Comparative Fit Index do modelo proposto com o nulo foi 0,98, o que mostra um bom ajuste e indica a aceitação do modelo. Nesse índice, quanto mais o valor se aproxima de 1,0, melhor o ajuste (Fife-Schaw, 2010).

O Goodness of Fit Index foi 0,97, e o Adjusted Goodness of Fit Index (AGFI, Índice Ajustado de Qualidade do Ajuste) foi 0,94. Esses índices representam a proporção da variância explicada através da estimação, e são esperados valores próximos a 1,0 como indicadores de bom ajuste (Fife-Schaw, 2010).

O alfa de Cronbach para a EAR foi 0,87, considerado muito bom. A média de correlação interitem foi 0,49, com um mínimo de 0,27 e um máximo de 0,74 . A correlação item-total variou de 0,38 a 0,81 .
Através do coeficiente de Pearson, a EAR apresentou correlação estatística significativa de 0,84 com os dois itens de satisfação amorosa de Jemmott III et al. (1989). Além disso, a EAR e a EAD total e suas subescalas - satisfação diádica, consenso diádico, coesão diádica e expressão de afeto - correlacionaram-se significativamente, apresentando os seguintes coeficientes: $0,81,0,80,0,67,0,57$ e 0,58 respectivamente. Em todos os casos, os níveis de significância estatística foram menores do que 0,01 .

Análises de variância não encontraram diferenças estatísticas significativas $(p<0,05)$ de satisfação no relacionamento entre homens e mulheres, entre coabitantes e não coabitantes e entre as condições de com filho e sem filho na relação. Além disso, não houve correlações estatísticas significativas entre satisfação no relacionamento e idade dos participantes ou tempo de duração da relação. Houve, porém, diferença significativa entre as médias de satisfação no relacionamento $(p<0,01)$ entre pessoas classificadas como ajustadas $(3,8)$ e desajustadas (2,3) conjugalmente na EAD segundo Spanier (1976).

Considerando apenas os escores dos participantes casados e em união estável ( $n=182)$, houve considerável aumento na magnitude das correlações estatísticas significativas da EAR com os dois itens $(r=0,87)$ de Jemmott III et al. (1989) e com a EAD total $(r=0,86)$ e suas subescalas: satisfação diádica $(r=0,83)$, consenso diádico $(r=0,76)$, coesão diádica $(r=0,55)$ e expressão de afeto $(r=0,68)$. Contudo, não foram encontradas diferenças estatísticas significativas em satisfação no relacionamento entre homens e mulheres e entre a condição com filho e sem filho; nesse último caso, diferentemente dos participantes paraibanos. As correlações entre a satisfação no relacionamento e a idade e o tempo de duração da relação dos participantes também não foram significativas.

\section{Discussão}

Segundo os resultados, a versão brasileira EAR apresentou uma estrutura fatorial coerente com a proposta original de Hendrick (1988) em 
ambos os estudos realizados. Esse desempenho psicométrico apresentou-se estável, com escores de amostras diferentes provenientes de culturas do Nordeste e do Sul do País. Na análise fatorial confirmatória, os índices relativos à adequação de modelos de equações estruturais forneceram razoáveis evidências da estrutura unifatorial da EAR.

Comparando os resultados encontrados no presente trabalho a estudos anteriores produzidos em diversas culturas (Hendrick et al., 1998; Dinkel \& Balck, 2005; Renshaw, McKnight, Caska, \& Blais, 2011; Vaughn \& Baier, 1999), notam-se grandes igualdades nos resultados e pequenas diferenças de valores que, indubitavelmente, replicam a estrutura unifatorial da EAR.

Com relação à consistência interna dos instrumentos, os elevados alfas de 0,89 e 0,87 nos estudos atuais I e II, respectivamente, igualam-se à média de 0,87 encontrada por Graham, Diebels e Barnow (2011) em estudo de meta-análise sobre a fidedignidade da EAR, com 398 artigos que representam 622 amostras e 636806 indivíduos, que forneceram 639 coeficientes alfas. Os autores afirmam que, em grande parte das pesquisas posteriores (por exemplo, Dinkel \& Balck, 2005), os escores obtidos tendem a superar os valores encontrados quando do desenvolvimento da EAR.

No estudo original, Hendrick (1988) encontrou elevados coeficientes de correlações entre a EAR e a EAD, especialmente na subescala satisfação diádica, sugerindo fortes evidências de validade convergente. Precisamente, os mesmos resultados foram achados por Vaughn e Baier (1999) em uma amostra clínica norte-americana e por Dinkel e Balck (2005) com os escores de uma versão alemã da EAR. Da mesma forma, no presente estudo, para os escores da versão brasileira da EAR, resultados semelhantes foram encontrados, gerando robustas evidências de validade convergente, haja vista que a validade dos escores da $E A D$, no Brasil, foi verificada por Hernandez (2008). Reforçando esses resultados, as pessoas ajustadas na EAD apresentaram médias de satisfação no relacionamento (EAR) maiores do que as classificadas como desajustadas. Além disso, foi encontrada elevada correlação da EAR com os itens

334 de satisfação global de Jemmott III et al. (1989).
Diferentemente do estudo II, no primeiro estudo deste trabalho, foi encontrada uma diferença estatística significativa em satisfação no relacionamento entre os sujeitos com e sem filhos. Nesse caso, a condição sem filhos apresentou níveis maiores de satisfação. Willi (1997) comentou que menor satisfação é mais marcadamente encontrada em casais com crianças, enquanto aqueles sem crianças apresentam escores mais altos. Dinkel e Balck (2005) também encontraram pessoas que não coabitavam e sem filhos com níveis mais elevados de satisfação no relacionamento. No entanto, análise dos escores dos sujeitos casados (com e sem filho) da amostra do estudo II, atual, não corroborara esses resultados. Sternberg (1989) explicou que a satisfação conjugal é elevada durante os primeiros anos da relação, declina durante os anos intermediários e volta a elevar-se nos últimos anos. Uma razão para esse modelo em forma de $U$ é, provavelmente, o efeito dos filhos. Está claro que a chegada dos filhos cria novas tensões e se associa a uma diminuição da satisfação conjugal (Hernandez \& Hutz, 2009). À medida que os filhos crescem e, por fim, saem de casa, a satisfação com o matrimônio pode melhorar.

Curiosamente, uma medida que foi originalmente desenvolvida com uma amostra de estudantes para medir satisfação em diversos tipos de relacionamentos apresentou desempenho psicométrico ótimo (fatorial, consistência interna e na relação com outras medidas) com pessoas casadas ou com união estável, conforme as análises do estudo I (amostra integral de casados e união estável) e estudo II (apenas com os casados e união estável). Na sua pesquisa, Graham et al. (2011) constataram essa situação no que tange à fidedignidade da EAR. Da mesma forma que o estudo atual, Dinkel e Balck (2005) não encontraram diferenças estatísticas significativas entre homens e mulheres, entre tipos de relacionamentos (casados, noivos e namorados) em satisfação no relacionamento medida pela EAR.

Além disso, Dinkel e Balck (2005) não encontraram relação entre a idade, a duração do relacionamento dos sujeitos e a satisfação no relacionamento, resultados que se repetiram na presente 
pesquisa. No entanto, os autores acharam correlação negativa significativa entre duração do relacionamento e os escores da EAR apenas para os não casados, o que não ocorreu no estudo atual.

Considerando a análise da estrutura fatorial da EAR, os resultados do presente estudo replicaram os achados de Hendrick (1988) e outros que, posteriormente, verificaram a estrutura da escala (Dinkel \& Balck, 2005; Hendrick et al., 1998; Renshaw et al., 2011; Vaughn \& Baier, 1999). Um fator geral emergiu das análises, o que demonstrou claramente a validade fatorial da EAR.

A Escala de Avaliação do Relacionamento, na versão atual, também é uma medida homogênea com elevada consistência interna. A correlação item-total é muito boa, comparável à pesquisa original (Hendrick, 1988) e à extensa meta-análise (Graham et al., 2011). Adicionalmente, houve uma correlação substancial entre a EAR e a EAD e suas subescalas, especialmente a satisfação diádica, evidências de convergência.

No conjunto, as evidências sugerem que a versão brasileira da EAR é uma medida confiável e válida, adequada para a avaliação subjetiva global dos relacionamentos íntimos. Contudo, algumas limitações estão presentes neste estudo e podem ter influenciado os resultados. As amostras de ambos os estudos não são probabilísticas e, embora possam representar culturas diversas, estão distantes de cobrir toda a diversidade existente no País, no entanto futuras investidas poderão preencher essas lacunas.

\section{Referências}

Andrade, A. L., Garcia, A., \& Cano, D. S. (2009). Preditores da satisfação global em relacionamentos românticos. Psicologia: Teoria e Prática, 11(3), 143-156.

Berscheid, E. (2010). Love in the fourth dimension. Annual Review of Psychology, 61, 1-25.

Boomsma, A. (2000). Reporting analyses of covariance structures. Structural Equation Modeling, 7(3), 461-483.

Burgess, E. W., \& Wallin, P. (1944). Predicting adjustment in marriage from adjustment in engagement. American Journal of Sociology, 49(4), 324-330.

Byrne, B. M. (2010). Structural equation modeling with Amos: Basic concepts, applications, and programming. New York: Routledge.
Dinkel, A., \& Balck, F. (2005). An evaluation of the German relationship assessment scale. Swiss Journal of Psychology, 64(4), 259-263.

Fife-Schaw, C. (2010). Introdução à modelagem de equação estrutural. In G. M. Breakwell, S. Hammond, C. Fife-Schaw, \& J. A. Smith (Eds.), Métodos de pesquisa em psicologia (pp.434-453). Porto Alegre: Artmed.

Fowers, B. J., Applegate, B., Olson, D. H., \& Pomerantz, B. (1994). Marital conventionalization as a measure of marital satisfaction: A confirmatory factor analysis. Journal of Family Psychology, 8(1), 98-103.

Glenn, N. D. (1990). Quantitative research on marital quality in the 1980s: A critical review. Journal of Marriage and the Family, 52, 818-831.

Graham, J. M., Diebels, K. J., \& Barnow, Z. B. (2011). The reliability of relationship satisfaction: A reliability generalization meta-analysis. Journal of Family Psychology, 25(1), 39-48.

Hair, J. F., Jr., Anderson. R. E., Tatham, R. L., \& Black, W. C. (2005). Análise multivariada de dados. Porto Alegre: Bookman.

Hancock, G. R., \& Freeman, M. J. (2001). Power and sample size for the root mean square error of approximation test of not close fit in structural equation modeling. Educational and Psychological Measurement, 61(5), 741-758.

Hendrick, S. S. (1981). Self-disclosure and marital satisfaction. Journal of Personality and Social Psychology, 40(6), 1150-1159.

Hendrick, S. S. (1988). A generic measure of relationship satisfaction. Journal of Marriage and the Family, 50, 93-98.

Hendrick, S. S., Dicke, A., \& Hendrick, C. (1998). The Relationship Assessment Scale. Journal of Social and Personal Relationships, 15, 137-142. doi: 10.1177/026 5407598151009

Hendrick, S. S., \& Hendrick, C. (1997). Love and satisfaction. In R. J. Sternberg, \& M. Hojjat (Eds.), Satisfaction in close relationships (pp.56-78). New York: The Guilford Press.

Hendrick, S. S., Hendrick, C., \& Adler, N. L. (1988). Romantic relationships: Love, satisfaction, and staying together. Journal of Personality and Social Psychology, 54(6), 980-988.

Hernandez, J. A. E. (2008). Avaliação estrutural da escala de ajustamento diádico. Psicologia em Estudo, 13(3), 593-601.

Hernandez, J. A. E., \& Hutz, C. S. (2009). Transição para a parentalidade: ajustamento conjugal e emocional. Psico, 40(4), 414-421.

Huston, T. L., Caughlin, J. P., Houts, R. M., Smith, S.E., \& George, L. J. (2001). The connubial crucible: Newlywed years as predictors of marital delight, distress, and divorce. Journal of Personality and Social Psychology, $80(2), 237-252$. 
Jemmott III, J. B., Ashby, K. L., \& Lindenfeld, K. (1989). Romantic commitment and the perceived availability of opposite-sex persons: On loving the one you're with. Journal of Applied Social Psychology, 19(14), 1198-1211.

Locke, H. J., \& Wallace, K. M. (1959). Short maritaladjustment and prediction tests: Their reliability and validity. Marriage and Family Living, 21(3), 251-255.

McDonald, R. P., \& Ho, M. R. (2002). Principles and practice in reporting structural equation analyses. Psychological Methods, 7(1), 64-82.

Magagnin, C., Körbes, J. M., Hernandez, J. A. E., Cafruni, S., Rodrigues, M. T., \& Zarpelon, M. (2003). Da conjugalidade a parentalidade: gravidez, ajustamento e satisfação conjugal. Aletheia, (17-18), 41-52.

Norgren, M. B. P., Souza, R. M., Kaslow, F., Hammerschmidt, H., \& Sharlin, S. A. (2004). Satisfação conjugal em casamentos de longa duração: uma construção possível. Estudos de Psicologia, 9(3), 575-584.

Patterson, G. R. (1976). Some procedures for assessing changes in marital interaction patterns. Research Bulletin, 16(7), 1-29.

Perlin, G., \& Diniz, G. (2005). Casais que trabalham e são felizes: mito ou realidade? Psicologia Clínica, 17(2), 15-29.

Prouty, A. M., Markowski, E. M., \& Barnes, H. L. (2000). Using the dyadic adjustment scale in marital therapy: An exploratory study. The Family Journal: Counseling and Therapy for Couples and Families, 8(3), 250-257.

Renshaw, K. D., McKnight, P., Caska, C. M., \& Blais, R. K. (2011). The utility of the relationship assessment scale in multiple types of relationships. Journal of Social and Personal Relationships, 28(4), 435-447.
Rusbult, C. E. (1983). A longitudinal test of the investment model: The development (and deterioration) of satisfaction and commitment in heterosexual involvements. Journal of Personality and Social Psychology, 45(1), 101-117.

Russel, R. J. H., \& Wells, P. A. (1992). Social desirability and quality of marriage. Personality and Individual Differences, 13(7), 787-791.

Snyder, D. K. (1979). Marital satisfaction inventory. Los Angeles: Western Psychological Services.

Scorsolini-Comin, F., \& Santos, M. (2010). Satisfação conjugal: revisão integrativa da literatura científica nacional. Psicologia: Teoria e Pesquisa, 26(3), 525-532.

Spanier, G. B. (1976). Measuring dyadic adjustment: New scales for assessing the quality of marriage and similar dyads. Journal of Marriage and the Family, 38(1), 15-28.

Sternberg, R. J. (1989). El triángulo del amor: intimidad, amor, compromiso. Barcelona: Ediciones Paidós.

Vaughn, M. J., \& Baier, M. E. M. (1999). Reliability and validity of the relationship assessment scale. The American Journal of Family Therapy, 27(2), 137-147.

Wachelke, J. F. R., Andrade, A. L., Cruz, R. M., Faggiani, R. B., \& Natividade, J. C. (2004). Medida da satisfação em relacionamento de casal. Psico-USF, 9(1), 11-18.

Willi, J. (1997). The significance of romantic love for marriage. Family Process, 36(2), 171-182.

Recebido em: 2/2/2012

Versão final em: 18/6/2012

Aprovado em: 12/7/2012 